\title{
Digital Image Forgery Detection using Correlation Coeficients
}

\author{
Chhaya Saini \\ M. Tech. Scholar \\ CSE Dept. KEC \\ Ghaziabad, India
}

\author{
Priya Singh \\ M. Tech. Scholar \\ CSE Dept. MIET \\ Meerut, India
}

\author{
Pramod Kr. Sethy \\ Assistant Professor \\ CSE Dept. KEC \\ Ghaziabad, India
}

\author{
Raj Kumar Saini \\ P.hd. Research Scholar \\ CSE Dept. IIT Roorkee \\ Roorkee, India
}

\begin{abstract}
In digital era, it has become easy to modify any image. Due to this the trust and validation of it is going to lose. Now it has become major problem of digital world to regain the lost trust. The background behind the modification and any changes in an image is easy availability of software tools on internet. Images can be transformed from one image format to another and any part of image can be altered pixel by pixel. Before the digital age, it was literally easy to detect the altered photographs. But now with the advent in the commercial software like various image photo editing software like Adobe Photoshop, XnView; ProShow Gold etc. make image forgery simple, the tampering of the photographs have become very easy, can be carried out without any noticeable signs of tampering and it is becoming harder to expose and mark the authentic ones. With the increased dependency over the digital images for exchanging the information, the need to keep their authenticity increases and digital images also use as authenticated facts for an offence. If it will not contain the authenticity then a problem will arise. An image forgery is made either by summing some templates, or hiding some kind of information in an image, in which the consistency is lost. This paper identifies the key methods for detecting forgery in the digital images. To identify and detect the forged areas, the image is divided into overlapped patches of some fixed size. In our paper we will discuss the correlation method, that how it find outs the forged part in an image. Firstly, the digital image tampering process is discussed. After that, it shows that different algorithms have different approaches to detect the forgery.
\end{abstract}

\section{Keywords}

Image Forgery; Mean Vector Method, Correlation Coefficient, Templates.

\section{INTRODUCTION}

In the present world which has become digital computing world, the exchanging and representing the information in visual manner has become more essential. Due to great evolution in digital computation and networking technologies, the earlier period have showed a significant hike in the accessibility, and broadcasting of digital images using digital image processing software's. However, manipulation and forgeries are also created by these technologies in digital images and due to this it become difficult to tell between original image and forged image. Forgery of images contains pasting one part of an image onto another image, expertly manipulated to avoid any notion. Each image changes may be a forgery based upon the perspective in which it is used. The advanced and inexpensive software of digital era enable the manipulation of digital images with undetectable hints. On an image, manipulation includes these processes like rotation, scaling, brightness changes, contrast enhancement, blurring, etc. or any combinations of them. Now it has become more complex to establish image authenticity and this problem is harder to sort out due to the availability of digital images and free image editing tools

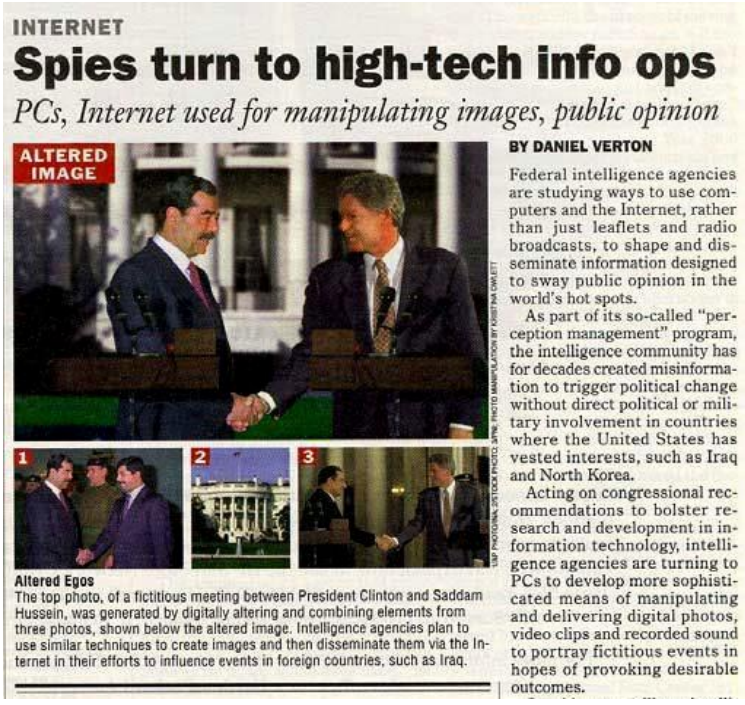

Fig 1: Example of a Digital Image Forgery

The Figure above shows a famous example of digital image forgery. In which a newspaper cutout shows, three different pictures were collected from different sources and merged together to create a forged image: Pictures of Saddam Hussein, The White House, and Bill Clinton. Here White House has been blurred to show a real effect a farther focus background. Then, the images of Mr. Bill Clinton and $\mathrm{Mr}$. Saddam Hussein were clipped from two distinct pictures and imposed on the White House. The image is forged with realistic effects, it care the correct shadows of speaker stands with microphones.

\section{IMAGE TAMPERING DETECTION TECHNIQUES}

The forgery detection techniques are divided into two types, Active approaches and Passive approaches. These approaches identify the forged digital images. In the active methods, we add some data or signature into the original image to keep it safe from forgery, but in passive methods we don't have original image, so we can't insert any data and we should perform operations on some features of the image e.g. on correlations, compressions, statistical anomalies and measurements of attributes in the given image to detect forgery. The passive approaches are subdivided into five categories. These are camera-based, format-based, pixelbased, geometric-based and physical-based. Active approaches can be divided into two types, the embedding position spatial domain or frequency domain data. 


\subsection{Active Approaches}

The Active approach considered to insert the data and signature into the original image at the resource side and make sure the image reliability at the detection side. Basically, Active approach have original image to embedding the data and signature into the image.

\subsection{Passive Approaches}

In Passive approaches, there is no availability of original images. It works in the absence of the data and signature. I use the attributes of an image to detect forgery in it. Passive approach is divided into five sub methods: pixel-based method, Formats-based method, Physically-based method, camera-based method, and Geometry- based method.

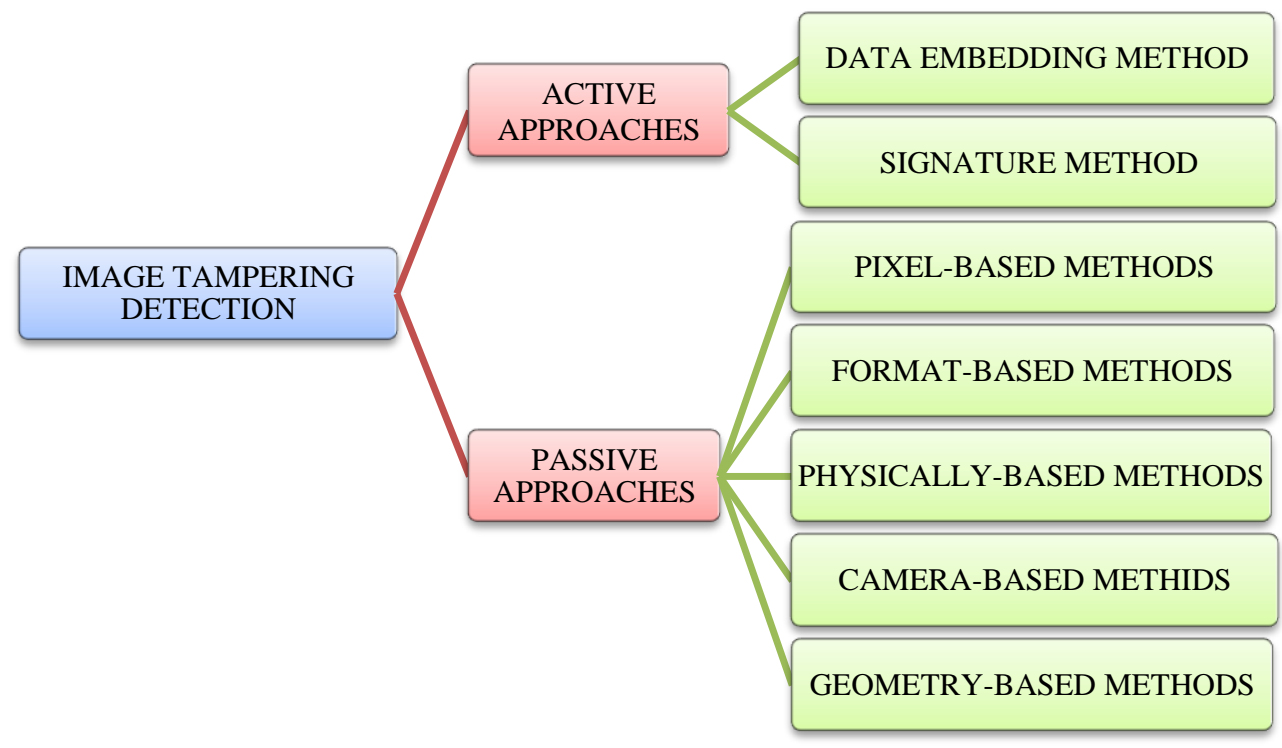

Figure 2(a): Digital image forgery detection approaches

\section{RELATED WORK}

In the field of digital image processing, a lot of work is done to detect the tampered images. There are so many techniques to create a forgery but copy move is one of easy and famous technique. In copy move forgery, one of the portions of the image is copied and moved to another part in the same image. Lots of methods are there to detect these types of forgeries. Fridrich et al. [1], recommended a technique to identify copymove tampering, it works on analyzing the image to each and every cyclic shifted version. Due to the high complexity, it needs $(\mathrm{mn})^{2}$ steps to execute an image of size $\mathrm{M} \times \mathrm{N}$. Due to the high complexity, it become typical to implement. Ashima Gupta1 et al [3] proposed the technique to detect the region duplication with the help of Discrete Cosine Transform (DCT). In the technique of DCT, the forgery is detected by dividing the image in the overlapping blocks and the duplicated blocks are identified. But it fails in small copied area to detect forged blocks. One more approaches Pradyuman Deshpandey in which there are two methods; here first algorithm works effectively for copy-move to detect the copied part (copied without any changes) at different region in same image. Second algorithm is failed to detect very tiny copied part and it can't handle rotated images.

Auto regressive coefficient as element vector and artificial neural network (ANN) classifier method is developed by the Gopi et al [5] to detect image tampering. In it, 300 attributes vectors were used (form different images) to train an ANN. Another 300 attributes vector used to test an ANN. The Hit percentage in this experiment to detect the forgery is $77.67 \%$ in which forged images were used to train ANN and 94.83\% in experiment in which a database of forged images was used. The forgery detection approach using $3 \mathrm{D}$ lighting system is given by Fan et al. [4], based on the shape by shading. It's a hopeful technique in detecting the forgery through 3D lighting system but problem with it is assessment of $2 \mathrm{D}$ figures of object leftover.

The process of detecting a copy move forgery is similar to the process of feature extraction. Other methods are also used and are currently worked on reducing dimensionality [2], [6], moments [7], [8] color properties [9], frequency domain transform [1].

\section{PROPOSED WORK}

There are two methods that can spy forgery in BMP images and then place the forged elements. Our methods detects forged region by partitioning the image into overlapping patches and then test for the forged area. The efficiency and robustness of this technique at realistic forgeries level has been computed in this paper. Here, it can be verified that the algorithm is efficient for noisy images too.

\subsection{Mean Vector Method}

From the given image figure 4(a) first we calculate two mean vectors namely row mean vector and column mean vector that may be calculated as follows. Let image has $\mathrm{M}$ rows and $\mathrm{N}$ columns, and then the row mean vector is calculated as.

$$
\begin{aligned}
& \text { Row Mean Vector }=\frac{1}{\mathrm{M}} \sum_{i=1}^{M} r i \\
& \text { Column Mean Vector }=\frac{1}{\mathrm{~N}} \sum_{j=1}^{N} C j
\end{aligned}
$$

Where $r_{i}$ and $c_{j}$ represent ith row and $j$ th column in the image. 


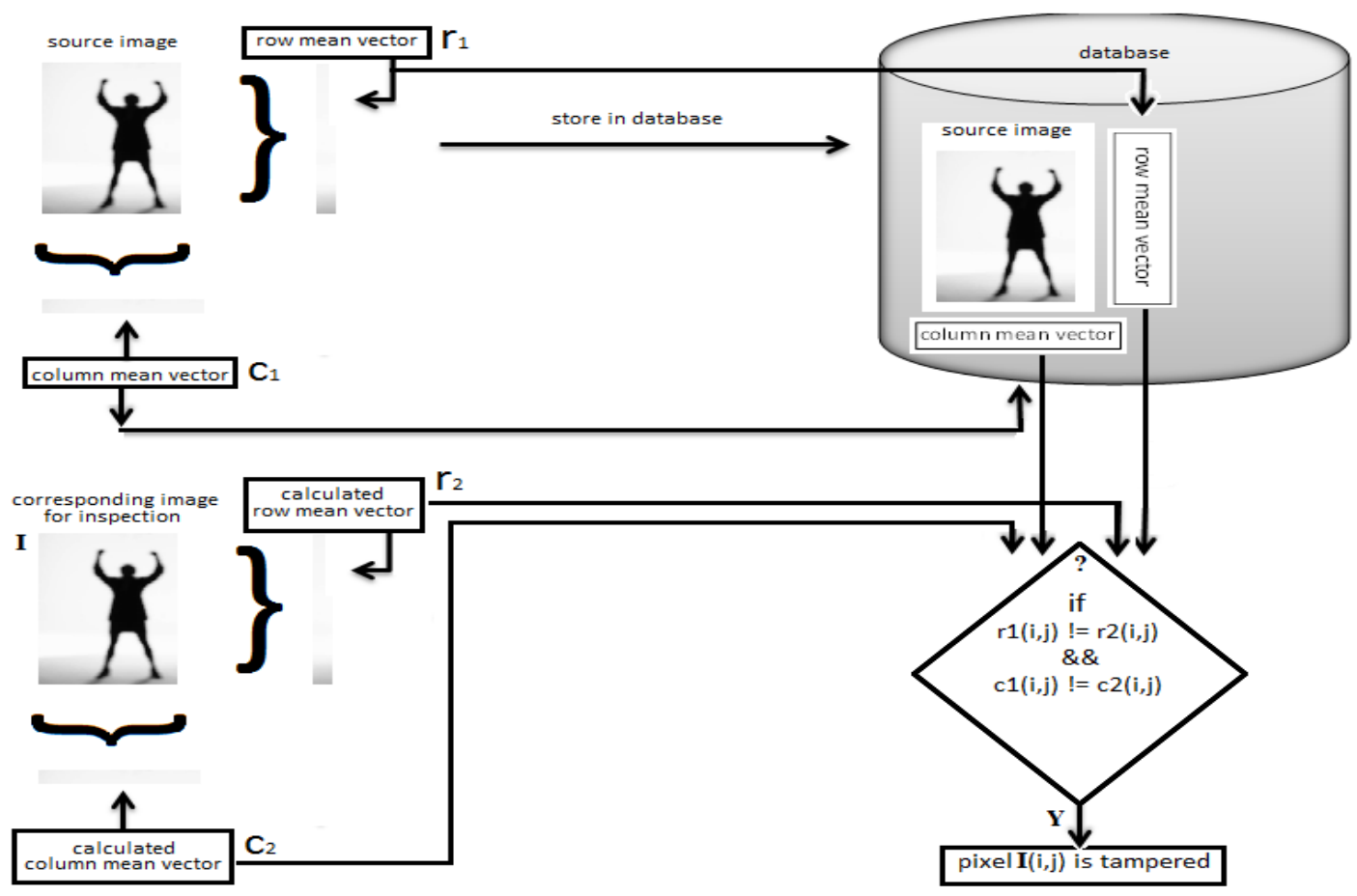

Fig 4(a): Mean Vector Testing

To identify the forgery, the Row and Column Mean Vector are calculated for both images, i.e., the Original image (r1, c1) and the Forged image (r2, c2). In case the image is forged, then these values for two images do not match up and then the pixel $I(i, j)$ is tampered in the image.

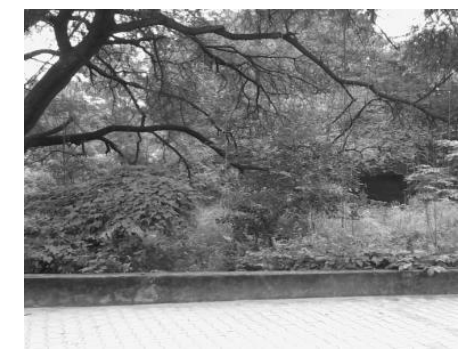

Fig 4(b): Original image

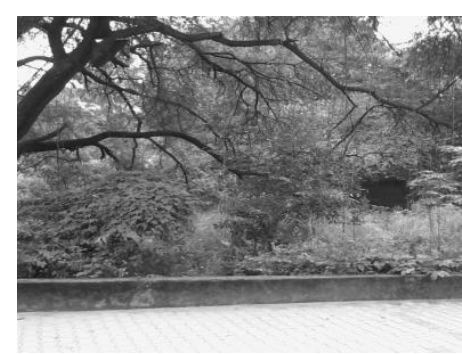

Fig 4(c): Forged image

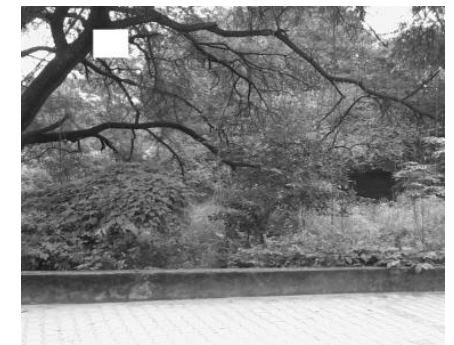

Fig 4(d): Result Image

In given example, Fig 4(b) is original image and Fig 4(c) is forged image in which some division of image is forged.

After comparing the row mean vector and column mean vector of both images it find out the tempered part in the image which showed in fig 4(d).

\subsection{Correlation Method}

Correlation method is used as a statistical tool to establish the association between two variables. The 2-D correlation is defined as follows:

$$
r=\frac{\sum_{m} \sum_{n}\left(\mathrm{~A}_{m n}-\overline{\mathrm{A}}\right)\left(\mathrm{B}_{m n}-\overline{\mathrm{B}}\right)}{\sqrt{\left(\sum_{m} \sum_{n}\left(\mathrm{~A}_{m n}-\overline{\mathrm{A}}\right)^{2}\right)\left(\sum_{m} \sum_{n}\left(\mathrm{~B}_{m n}-\overline{\mathrm{B}}\right)^{2}\right)}}
$$

1. Where, $r$ varies within the range -1 and 1 i.e. $-1 \leq r \leq_{1}$

2. Where A and B are 2-D data set and,

3. $\overline{\mathrm{A}}, \overline{\mathrm{B}}$ are means of sets $\mathrm{A}$ and $\mathrm{B}$ respectively.

4. $\mathrm{m}=1,2,3, \ldots, \mathrm{M}$ and $\mathrm{n}=1,2,3, \ldots, \mathrm{N}$. 


\section{5. $\mathrm{M} \times \mathrm{N}$ is size of $\mathrm{A}$ and $\mathrm{B}$.}

Here the whole original image having dimension $M * N$ is partitioned into the smaller overlapping blocks of dimension $m * n$. Thus the total no. of blocks will be $(M-$ $m+1) *(N-n+1)$. After partition an image into blocks, the correlation coefficients were calculated through above given formula between the adjacent overlapping blocks. This experiment is done at source side (on original image) and then same formula is applied on the destination side (on forged image). There is a threshold value 0.025 to establish the forgery level between two images. All adjacent blocks are traced to calculate the values of correlation coefficient for both images (original image and forged image) and take the difference of value of corresponding correlation coefficients from original and forged image.

If calculated correlation coefficient is greater than the threshold value 0.025 , then there is forgery in an image.

In case of working with two 1-D data set, the 1-D correlation may be defined as follows:

$$
r=\frac{\sum_{i}(\mathrm{~A} i-\overline{\mathrm{A}})(\mathrm{B} i-\overline{\mathrm{B}})}{\sqrt{\sum_{i}(\mathrm{~A} i-\overline{\mathrm{A}})^{2} \sum_{i}(\mathrm{~B} i-\overline{\mathrm{B}})^{2}}}
$$

The correlation calculation for two 1-D data set can be find out by putting the values of these data set in the given above formula. The value of $r$ should be varies within the range -1 and 1 . If value of $r$ is greater or less than the given interval then there is no correlation between them.

\section{EXPERIMENTAL RESULTS}

Here a mask or blocks of size $2 \times 2,4 \times 4,6 \times 6,8 \times 8,10 \times 10$, $12 \times 12,14 \times 14$ and $16 \times 16$ was taken and relative output images have been generated that are showing the sign of forgery in the digital images.

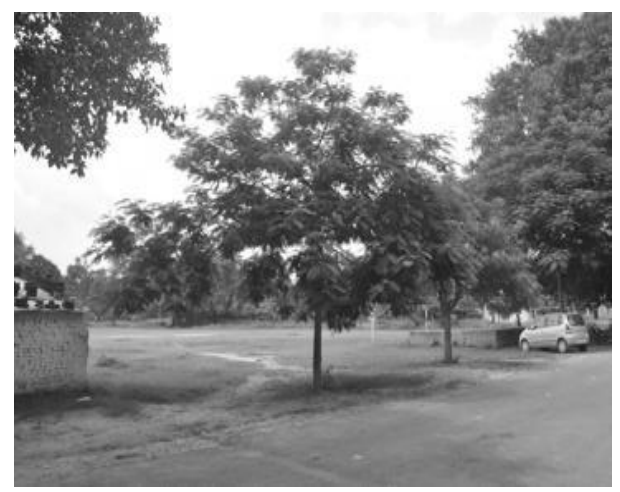

Fig 5(a): Original image

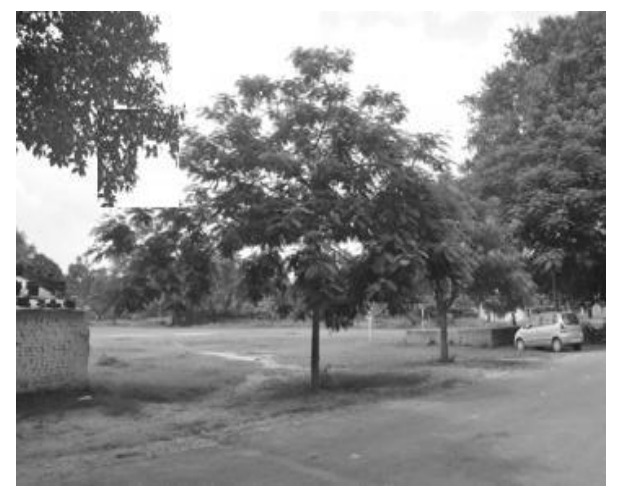

Fig 5(b): Forged Image

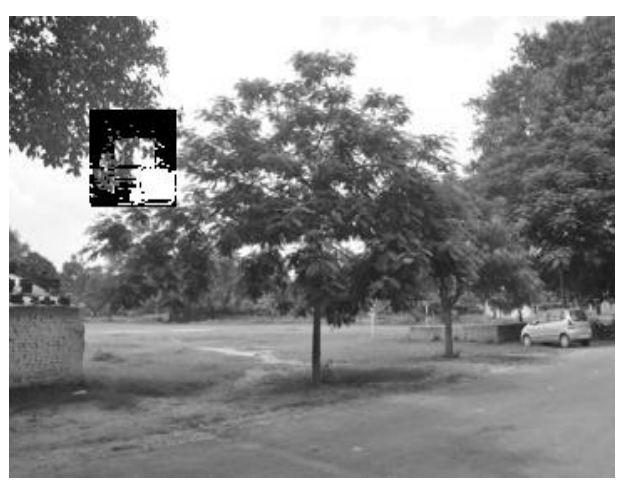

Fig 5(c): Result image $(2 \times 2)$

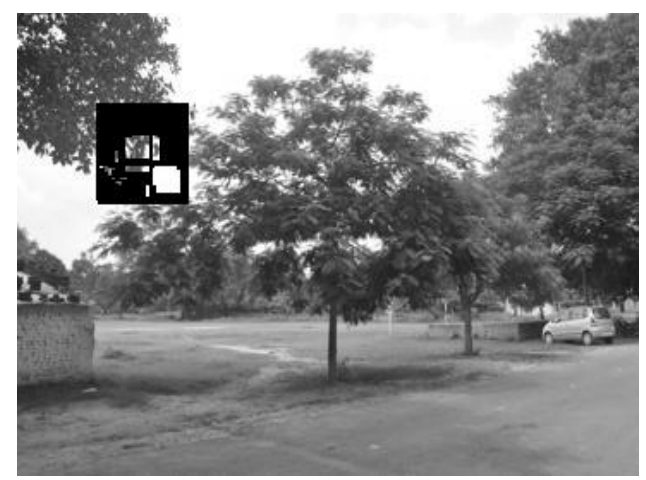

Fig 5(d): Result image $(4 \times 4)$

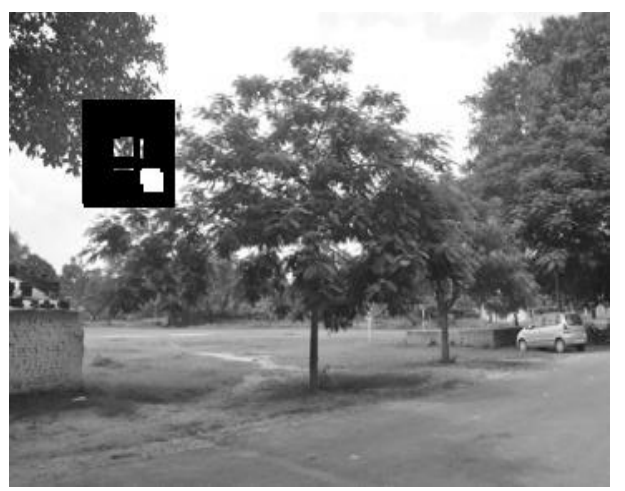

Fig 5(e): Result image $(6 \times 6)$

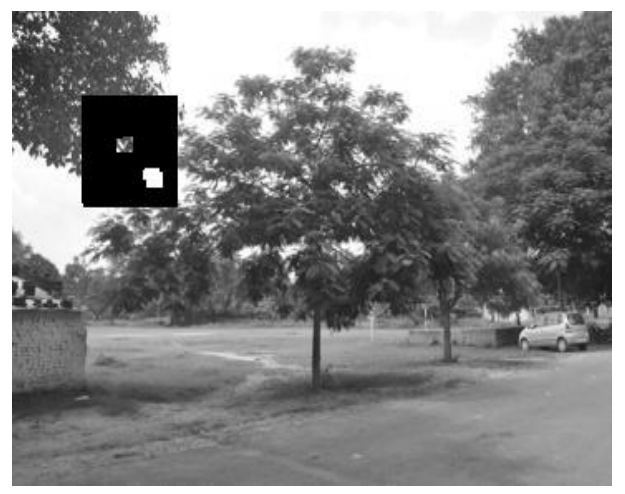

Fig 5(f): Result image $(8 \times 8)$ 


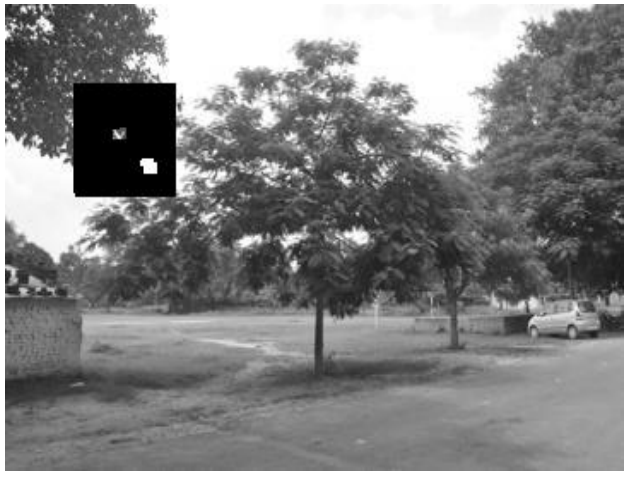

Fig 5(g): Result image $(10 \times 10)$

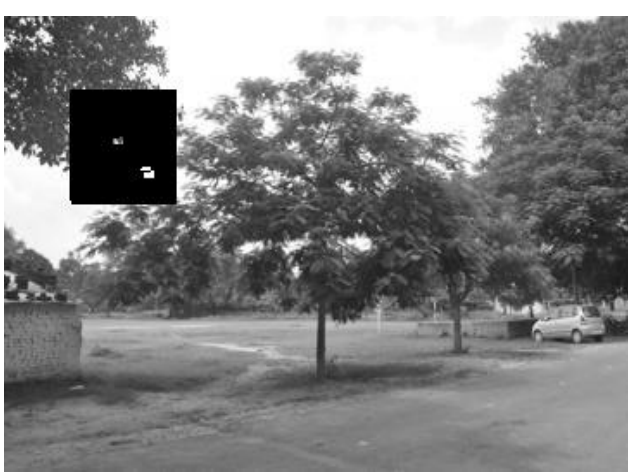

Fig 5(h): Result image $(12 \times 12)$

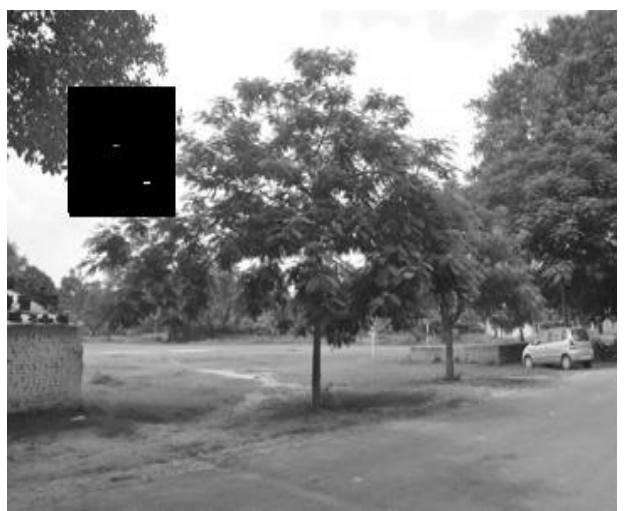

Fig 5(i): Result image $(14 \times 14)$

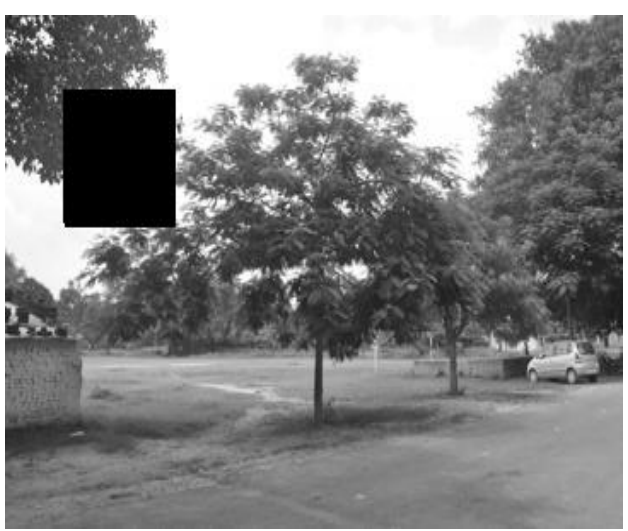

Fig 5(j): Result image $(16 \times 16)$
In the observed results if the mask size is increased the fault accepts increases and fault reject decreases.

\subsection{Data Base Preparation}

To accomplish the research work, a database of digital images is required. For this a word processing software for documentation of work is required .The database should be highly quality and scalable. Thus, an arrangement of collected and stable scene images was gathered to work upon. BMP image format can be preferred because it is simplest image format that directly store the intensity at each pixel in the image. It does not require compression technique. The database was collected and it covered mostly greenery and landscape images. Major work was done on MATLAB, some work was done on MS-paint and trail version of Adobe Photoshop cs2.We clicked more than 2000 digital images with different zooming using Nikon 16MP camera. It took around 6 months in collecting all data. Removal of noisy and those images those were very poor in terms of visibility of objects in the images.

Brightness is raised just by adding a constant " $\mathrm{K}$ " to the image.

$I^{\prime}=I+K$

Intensity normalization for making the dynamic range identical

$I^{\prime}=(I-a) \frac{(d-c)}{(b-a)}+c$

Where $(a, b)$ current dynamic is range and $(c, d)$ is new dynamic range.

$I$ and $I$ are current and new image.

\subsection{Experiment Configuration}

To execute the program, one needs a dual core (32-bit) machine with $3.2 \mathrm{GHz}$ processor speed using $1 \mathrm{~GB}$ of DDR2 Ram. MATLAB 7.0.1 is used to run the research as MATLAB software is used for all Algorithm coding. Extension is Bmp. All images are in color (RGB) and also converted into grayscale, Binary. Image resolution (Dimension) is $1600 \times 1600,242 \times 242$. Tampered shape Square $100 \times 100,50 \times 50$ pixels. Here one can take a $50(1600 \times 1600)$ $+53(242 \times 242)$ original images and $50(1600 \times 1600)$ $+53(242 \times 242)$ Tampered images. Number of forged region in image one. Camera used to take the pictures is Nikon 16MP camera. 


\subsection{Result Analysis}

TABLE 1. Comparison of Avg. False reject and False Accept with different block sizes (using Correlation coefficient)

\begin{tabular}{|l|l|l|l|l|l|l|l|l|l|}
\hline Mask Size & $\mathbf{2 \times 2}$ & $\mathbf{4 \times 4}$ & $\mathbf{6 \times 6}$ & $\mathbf{8 \times 8}$ & $\mathbf{1 0 \times 1 0}$ & $\mathbf{1 2 \times 1 2}$ & $\mathbf{1 4 \times 1 4}$ & $\mathbf{1 6 \times 1 6}$ & Average \\
\hline $\begin{array}{l}\text { Average } \\
\begin{array}{l}\text { Reject per } \\
\text { mask }\end{array}\end{array}$ & 223.8019 & 118.3396 & 88.66038 & 68.12264 & 53.79245 & 43.45283 & 34.51887 & 26.53774 & 82.1533 \\
\hline $\begin{array}{l}\text { Average } \\
\text { accept per } \\
\text { mask }\end{array}$ & 164.3028 & 245.884 & 368.7748 & 506.166 & 655.0569 & 810.1698 & 971.5783 & 1142.328 & 608.0326 \\
\hline
\end{tabular}

TABLE 2. Time analysis for each block $/$ mask size (each block =53 sets)

\begin{tabular}{|l|l|l|l|l|l|l|l|l|}
\hline Mask Size & $\mathbf{2} \times \mathbf{2}$ & $\mathbf{4 \times 4}$ & $\mathbf{6} \times \mathbf{6}$ & $\mathbf{8} \times \mathbf{8}$ & $\mathbf{1 0 \times 1 0}$ & $\mathbf{1 2 \times 1 2}$ & $\mathbf{1 4 \times 1 4}$ & $\mathbf{1 6 \times 1 6}$ \\
\hline $\begin{array}{l}\text { Average } \\
\text { Time per } \\
\text { mask }\end{array}$ & $\begin{array}{l}20.5263 \\
\mathrm{sec}\end{array}$ & $20.8106 \mathrm{sec}$ & $19.3596 \mathrm{sec}$ & $15.6688 \mathrm{sec}$ & $\begin{array}{l}20.3119 \\
\mathrm{sec}\end{array}$ & $15.6546 \mathrm{sec}$ & $\begin{array}{l}15.6728 \\
\mathrm{sec}\end{array}$ & $\begin{array}{l}14.8580 \\
\mathrm{sec}\end{array}$ \\
\hline
\end{tabular}

\subsection{Algorithm Efficiency}

Observing the table of Time analysis, it can be concluded that the time difference among the block sizes is minor. The forgery detection in each block size is approximately same.
In Fig 5(k), it can be seen that as block size increases the false reject is decreases and false accept is increases. Thus, large block size should be used to detect the forgery correctly.

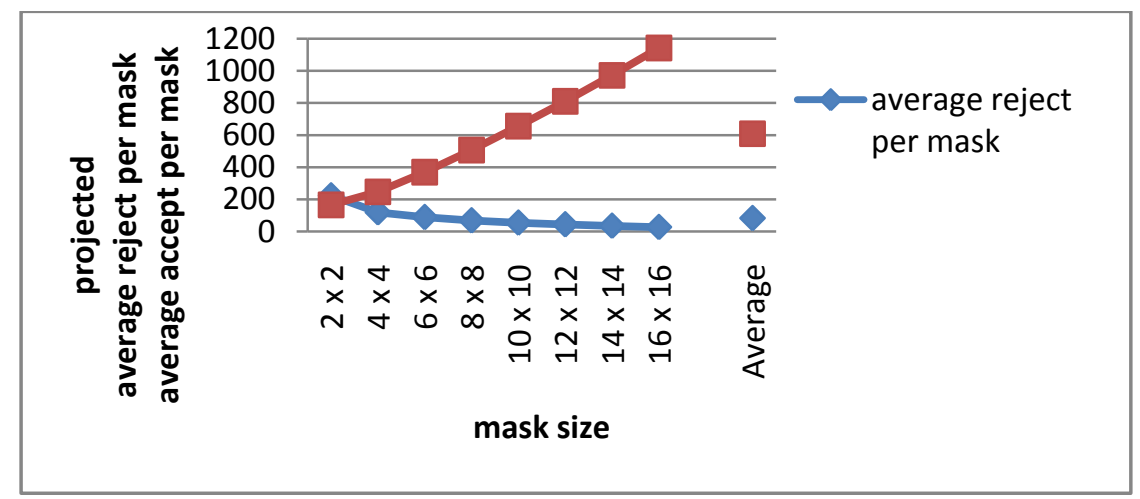

Fig 5(k): Graph shows False reject per mask size and false accept per mask size

This graph Fig 5(1) shows that the false reject is almost minor, near to zero for all sets ( There were 53 sets in the experiment
) and false accept is higher and same for all sets as per the blocks

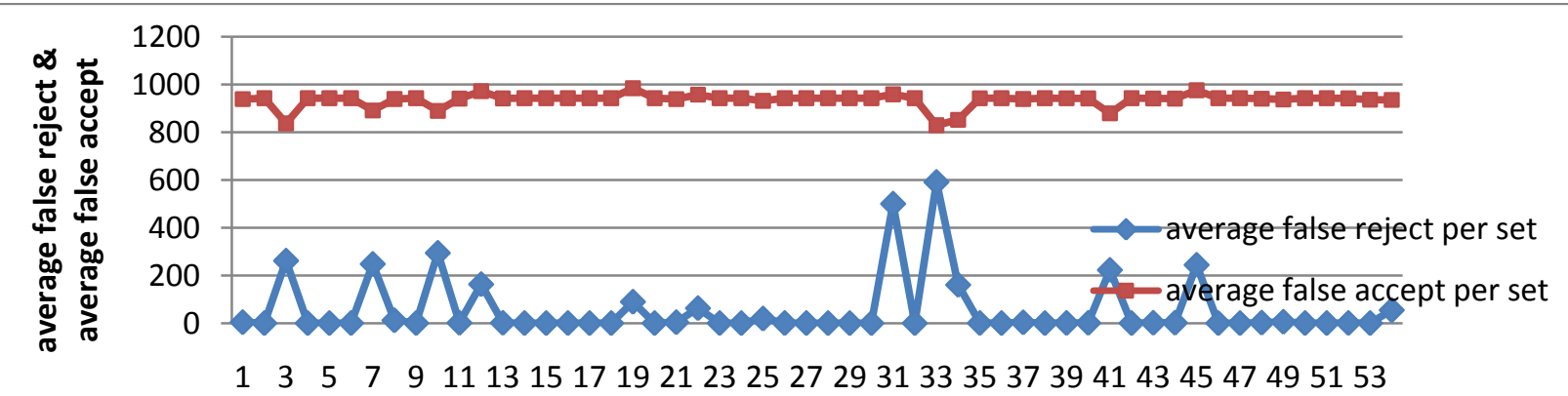

Set No.

Fig 5(1): Graphs shows false reject and false accept per set number 


\section{CONCLUSION}

Digital image forgery has become a common technique and is amongst the top most forgeries carried out in the current era. This report establishes what exactly the digital image forgery is. Some of the major approaches for digital image authentication and forgery detection are defined. The method described in this image is a robust approach to find out the forged part of an image. Here, in this bmp images have been used for dissertation. Mean Vector method works well with grey as well as with binary images and detects the forged region. Some tests were performed on the algorithm over the images to check our results.Correlation method detects forgery with some false acceptances and some false rejections. The experiment results in improved detection rate in forgery and also improved the detection time of the Digital image forgery hit uncovering algorithm that is used. Future work is to mature the second method and to produce better result. With more than one forged region in the image. With more than one and complex, irregular shapes of forged region like circle, ellipse, convex hull etc., improving the running time of technique.

\section{REFERENCES}

[1] J. Fridrich, D. Soukal, and J. Lukas, "Detection of CopyMove Forgery in Digital Images", in Proceedings of Digital Forensic Research Workshop, August 2003.

[2] C. Popescu and H. Farid, "Exposing Digital Forgeries by Detecting Duplicated Image Regions," Technical Report, TR2004-515, Department of Computer Science, Dartmouth College, pp. 758-767, 2006.
[3] Ashima Gupta , Nisheeth Saxena , S.K Vasistha, "Detecting Copy move Forgery using DCT", International Journal of Scientific and Research Publications, Volume 3, Issue 5, May 20131 ISSN 22503153

[4] W. Fan, K. Wang, F. Cayre and Z. Xiong, "3D LightingBased Image Forgery Detection Using Shape-FromShading", 20th European Signal Processing Conference EUSIPCO, (2012), pp. 1777-1781.

[5] E. Gopi, N. Lakshmanan, T. Gokul, S. Ganesh and P. Shah, "Digital image forgery detection using artificial neural network and auto regressive coefficients", Proc. Canadian conference on electrical and computer engineering, (2006), pp. 194-7.

[6] X. Kang and S. Wei, "Identifying Tampered Regions Using Singular Value Decomposition in Digital Image Forensics," International Conference on Computer Science and Software Engineering, pp. 926-930, 2008.

[7] B. Mahdian and S. Saic, "Detection of copy-move forgery using a method based on blur moment invariants.," Elsevier Forensic Science International, vol. 171, no. 2-3, pp. 180-189 Sep. 2007.

[8] S.-jin Ryu, M.-jeong Lee, and H.-kyu Lee, "Detection of Copy-Rotate- Move Forgery Using Zernike Moments," IH, LNCS 6387, vol. 1, pp. 51-65, 2010.

[9] W. Luo, J. Huang, and G. Qiu, "Robust Detection of Region-Duplication Forgery in Digital Image," 18th International Conference on Pattern Recognition (ICPR'06), pp. 746-749, 2006. 